Preprint typeset in JHEP style. - HYPER VERSION

RUNHETC-2001-01

hep-th/0101016

\title{
Supergravity Couplings of Noncommutative D-branes
}

\author{
Hong Liu* and Jeremy Michelson ${ }^{\dagger}$ \\ New High Energy Theory Center \\ Rutgers University \\ 126 Frelinghuysen Road \\ Piscataway, NJ 08854 USA
}

\begin{abstract}
We discuss the supergravity couplings of noncommutative D-branes by considering the disk amplitudes with one closed string insertion. The result confirms a recent proposal for the general form of the noncommutative Yang-Mills operators coupling to the massless closed string modes. The construction involves smearing Yang-Mills field variables along an open Wilson line. For multiple D-branes interacting with background supergravity fields, this prescription reduces, in the $B=0$ limit, to the "symmetrized trace" prescription, and the supergravity fields are seen to be functionals of the nonabelian scalar fields on the branes.
\end{abstract}

*liu@physics.rutgers .edu

${ }^{\dagger}$ jeremy@physics.rutgers.edu 


\section{Contents}

1. Introduction 1

2. Disk amplitudes with one closed string insertion

2.1 Preliminaries 4

2.2 One closed and one open 7

2.3 One closed and two open 8

3. Supergravity Couplings of Noncommutative D-branes 11

$3.1 \quad B=0$

3.2 Relation with Matrix Theory 15

4. The structure of higher-point amplitudes and the open Wilson line 16

5. Discussion and Conclusions 20

\section{Introduction}

It has become clear that open Wilson lines [1, 2] play a fundamental role in the dynamics of noncommutative gauge theories. They are essential to building gauge invariant operators and are thus essential when coupling noncommutative Yang-Mills modes to closed string modes [3, 4. They are also important for a proper understanding of the loop dynamics of noncommutative gauge theories, and of the Seiberg-Witten map [5] between ordinary and noncommutative Yang-Mills modes [6] (see also [7]).

An open Wilson line of momentum $k$ along some open contour $C$ is given by

$$
W_{k}(C)=\operatorname{Tr} \int d^{d} x W(x, C) * e^{i k \cdot x}
$$

where $W(x, C)$ is a Wilson line in which multiplication is given by the $*$-product. $W_{k}(C)$ is gauge invariant [1, 2] when the momentum $k$ of the line, and the distance $\Delta x$ between its two end points, satisfy the relation

$$
\Delta x^{\mu}=\theta^{\mu \nu} k_{\nu}
$$


where $\theta$ is the noncommutative parameter of the theory. Similarly, for any local operator $\mathcal{O}(x)$ which transforms adjointly under gauge transformations, $\mathcal{O}(k)$, defined by $^{1}$

$$
\mathcal{O}(k)=\operatorname{Tr} \int d^{d} x P_{*}[W(x, C) \mathcal{O}(y)] * e^{i k \cdot x},
$$

is gauge invariant [四], where $y$ is any point on the path $C$ of the Wilson line. Thus whereas there is no gauge invariant local operator in position space, it is possible to write down the linearized coupling of a supergravity field $h$ to the noncommutative Yang-Mills modes in momentum space [3, 四]

$$
\int d^{d} k h(-k) \mathcal{O}(k)
$$

with $\mathcal{O}(k)$ an operator of type (1.3).

However, since inserting local operators at any point of an open Wilson line all yields gauge invariant operators, it is not a priori clear what the precise ordering prescription is for constructing such a gauge invariant operator. In [6], by considering the factorization of the one-loop open string amplitudes [8, 9], it was proposed that the correct prescription is to integrate all external insertions over a straight Wilson line $e^{2}$. For example, the Yang-Mills operator that couples to the dilaton contains

$$
\int d^{d} x \int_{0}^{1} d \tau_{1} d \tau_{2} P_{*}\left[W(x, C) F_{\mu \nu}\left(x+\xi\left(\tau_{1}\right)\right) F^{\mu \nu}\left(x+\xi\left(\tau_{2}\right)\right)\right] * e^{i k \cdot x}
$$

with $C$ parameterized by $\xi(\tau)$, a straight line. In [10] it was argued based on the connection between noncommutative gauge theories and the Matrix model (see e.g. 11, 12, 13, 14, 14, 2, 15]) that the integration procedure provides a natural generalization to $U(\infty)$ of the "symmetrized trace" prescription of the Born-Infeld coupling to weak external supergravity fields (see e.g. [16, 17, 18]).

In this paper we investigate the disk amplitudes between one massless closed string in the NSNS sector and the noncommutative gauge modes on the brane, aiming to provide further support for the integration prescription and to write down the precise couplings of the massless closed string modes to a noncommutative D-brane. We will address RR insertions in a forthcoming paper. 119] We shall be interested in the leading terms of the amplitudes in the low energy limit in terms of open string metric $G_{\mu \nu}$ on the D-brane, while exploring the effect of the noncommutative parameter $\theta$. More explicitly, as in [8], we take the low energy limit to mean

$$
\alpha^{\prime} k_{i \mu} G^{\mu \nu} k_{j \nu} \ll 1 \quad \text { with } \quad k_{i \mu} \theta^{\mu \nu} k_{j \nu} \sim O(1) .
$$

\footnotetext{
${ }^{1} P_{*}$ denotes path ordering in which multiplication given by the $*$-product.

${ }^{2}$ Straight Wilson lines were first advocated in 四.
} 
Since the closed string metric $g_{\mu \nu}$ scales differently with respect to $\alpha^{\prime}$ from the open string metric, in the above limit (1.6), low energy processes in terms of the open string metric on the brane do not correspond to low energy processes in the bulk that involves the closed string metric. This differs from the $B=0$ behaviour.

The disk amplitudes of one massless closed string mode and two open string modes were previously given in [20, 21]. In particular in [21 (see also [22]) the low energy effective action in the limit (1.6) was found and compared with the Born-Infeld action. In this paper, with the role of an open Wilson line in mind, we show that the effective action can be written in a simpler and physically more transparent way than presented in [20, 21]. The result also confirms the integration prescription (1.5) proposed in [6, 10].

Physically, the appearance of the Wilson line and the integration prescription can be understood from the "stretched string" effect discussed in [23]. Recall that when $B=0$, the low energy external open string modes are point particles and the spacetime image of the worldsheet boundary is a single point, thus yielding local couplings between the closed string and gauge theory modes. With a nonzero $B$-field, the low energy open string behaves like an electric dipole that is in the presence of a strong background magnetic field [24, 25, 26]. As a result, an open string mode (even on-shell) has a finite spacetime extension $\Delta x^{\mu}=\theta^{\mu \nu} k_{\nu}$ [24, 23] proportional to its center of mass momentum. These open strings interact by splitting and joining their ends. When a closed string mode scatters off open string modes on a D-brane, all external open string modes join together to form a macroscopic open Wilson line, which then couples to the closed string mode. The extension of the Wilson line is given by

$$
\Delta x^{\mu}=\sum_{a} \Delta x_{a}^{\mu}=\theta^{\mu \nu} \sum_{a} k_{a \nu}=-\theta^{\mu \nu} q_{\nu}
$$

where $k_{a}$ and $q$ are the momenta of open and closed string modes respectively. From the worldsheet point of view, the Wilson line can then be viewed as the spacetime image of the worldsheet boundary, and the integration procedure has its origin in the integrations of the vertex operator insertions on the boundary worldsheet. It is interesting that in the noncommutative theory, the spacetime image of the worldsheet boundary, which is closed, is an open contour instead of a closed loop.

The way in which we write the string amplitudes previously given in [20, 21] makes it easy to extract the Wilson line from the operators $\mathcal{O}(k)$. We will also see that the effective couplings illustrate to all orders the expectation that when multiple D-branes interact with background supergravity fields, the supergravity fields should be regarded not just as functions of the $(p+1)$-dimensional spacetime but also as functionals of the nonabelian scalar fields (see e.g. [27, 17, 28, 18, 22]). For example, the coupling of a 
$D p$-brane to the graviton can be written as

$$
\int d^{p+1} x \operatorname{STr}\left[h_{\mu \nu}(X(x), x) T^{\mu \nu}(x)\right]
$$

where $X$ are the nonabelian adjoint scalar fields of the D-branes and $h_{\mu \nu}$ is the perturbation of the graviton. ${ }^{3}$ We also confirm to all orders the "symmetrized trace" prescription for the ordering in (1.8) up to terms quadratic in the field strength in $T^{\mu \nu}$.

This paper is organized as follows. In section 2 we present the string amplitudes for the interaction of a closed string with one or two open strings. From this result, we extrapolate, in section 3 an action which involves the open Wilson line. In section 1 we prove the proposal of section 4 by extracting the contribution to the Wilson line from the infinite series of disk diagrams with one closed string and an arbitrary number of open strings. This confirms the presence of the open Wilson line, in the postulated form. We conclude in section 5 with a discussion of the relation to Born-Infeld action.

As this work was being completed, ref. [30] appeared. That paper overlaps with this one. In particular, their energy momentum tensor agrees with the large $B$ limit of ours.

\section{Disk amplitudes with one closed string insertion}

\subsection{Preliminaries}

We will use $M, N=0,1, \ldots, 9$ to denote the spacetime indices; $\mu, \nu=0,1, \ldots, p$ to denote the worldvolume directions of a D-brane; and $i, j=p+1, \ldots, 9$ are the directions transverse to the brane. The relations [5] between the closed (open) string metric $g$ $(G)$ and coupling $g_{s}\left(G_{s}\right)$, the $B$-field, and the noncommutativity parameter $\theta$ are

$$
G^{-1}+\frac{\theta}{2 \pi \alpha^{\prime}}=\frac{1}{g+B}
$$

and

$$
G_{s}=g_{s}\left(\frac{\operatorname{det} G}{\operatorname{det} g}\right)^{\frac{1}{4}}
$$

We assume that $B$ lies only in Neumann directions (that is, along the $D$-brane) and that $g_{M N}$ vanishes for mixed Neumann/Dirichlet directions. Therefore the above equations apply to all spacetime directions; in particular, $G_{i j}=g_{i j}$ and $\theta^{i j}=0$.

\footnotetext{
${ }^{3}$ We use the coupling (1.8) to define the energy-momentum tensor, $T^{\mu \nu}$, of the noncommutative theory; this seems to differ from the Noether definition 29.
} 
The NSNS vertex operators are given by

$$
\begin{aligned}
V_{N S N S}^{-1,-1} & =\frac{2 g_{c}}{\alpha^{\prime}} e^{-\phi-\tilde{\phi}} e_{M N} \psi^{M} \tilde{\psi}^{N} e^{i q \cdot X} \\
V_{O}^{0} & =a_{M}\left(i \dot{X}^{M}+4 k \cdot \Psi \Psi^{M}\right) e^{i k \cdot X}
\end{aligned}
$$

where the overdot in equation (2.4) is understood to be the tangential derivative along the worldsheet boundary for Neumann directions, and the normal derivative for Dirichlet directions. The gauge bosons have momenta $k_{a}$ and polarizations $a_{a}$, with $a, b, \ldots$ labeling the gauge boson ${ }^{4}$; the massless closed string mode has momentum $q$ and polarization $e_{M N}$. We can decompose the closed string polarization into modes corresponding to perturbations of the graviton, $h_{M N}$, the antisymmetric $B$-field $b_{M N}$ and the dilaton $\varphi$ via

$$
e_{M N}=h_{M N}+b_{M N}+\frac{1}{2} \varphi\left(g_{M N}-q_{M} \rho_{N}-q_{N} \rho_{M}\right)
$$

In addition, $h_{M N}$ is traceless- $h_{M N} g^{M N}=0$ - and both $h_{M N}$ and $b_{M N}$ are transverse: $h_{M N} g^{N P} q_{P}=0=b_{M N} g^{N P} q_{P}$. Note that we exclusively use the closed string metric when discussing closed string fields. The auxiliary vector $\rho_{M}$ obeys $q_{M} g^{M N} \rho_{N}=1$ and is introduced to enforce transversality of $e_{M N}$,

$$
q_{M} g^{M N} e_{N P}=0=e_{P N} g^{N M} q_{M}
$$

but it will not appear in any total amplitudes. Also, momentum conservation requires $q_{\|}=-k \equiv-\sum_{a} k_{a}$, where $q_{\|}$and $q_{\perp}$ denote the components of $q$ parallel and perpendicular to the brane. In addition to (2.6), the on-shell conditions require that $q_{M} g^{M N} q_{N}=0=k_{a \mu} G^{\mu \nu} k_{a \nu}$ and $k_{a \mu} G^{\mu \nu} a_{a \nu}=0$.

We shall take the worldsheet to be the upper half plane and use the doubling trick to relate the the fields in the anti-holomorphic sector to those in the holomorphic sector

$$
\begin{aligned}
\tilde{X}^{\mu}(\bar{z}) & =\left(\frac{1}{g-B}(g+B)\right)^{\mu}{ }_{\nu} X^{\nu}(\bar{z}) \\
\tilde{X}^{i}(\bar{z}) & =-X^{i}(\bar{z})
\end{aligned}
$$

and

$$
\begin{aligned}
\tilde{\psi}^{\mu}(\bar{z}) & =\left(\frac{1}{g-B}(g+B)\right)^{\mu}{ }_{\nu} \psi^{\nu}(\bar{z}), & \Psi^{\mu} & =\frac{1}{2}\left(\psi^{\mu}+\tilde{\psi}^{\nu}\right)=\left(\frac{1}{g-B} g\right)^{\mu}{ }_{\nu} \psi^{\nu}, \\
\tilde{\psi}^{i} & =-\psi^{i}, & \Psi^{i} & =\frac{1}{2}\left(\psi^{i}-\tilde{\psi}^{i}\right)=\psi^{i} .
\end{aligned}
$$

\footnotetext{
${ }^{4}$ We have absorbed a factor of $g_{Y M}$ into the polarization. Also, we will suppress Chan-Paton factors from the formulas for simplicity, although we will comment on them when appropriate.
} 
$\Psi^{M}$ is the open string supersymmetric partner of $X^{M}$ which lives on the worldsheet boundary. By introducing $D=\operatorname{diag}(1,1, \ldots,-1, \ldots,-1)$-an identity matrix in the Neumann directions and minus the identity matrix in the Dirichlet directions - the above equations can be written collectively as

$$
\begin{aligned}
\tilde{X}^{M}(\bar{z}) & =\left(\frac{1}{g-B}(g+B) D\right)^{M}{ }_{N} X^{N}(\bar{z}), \\
\tilde{\psi}^{M}(\bar{z}) & =\left(\frac{1}{g-B}(g+B) D\right)^{M}{ }_{N} \psi^{N}(\bar{z}), \\
\Psi^{M} & =\left(\frac{1}{g-B} g\right)^{M}{ }_{N} \psi^{N}
\end{aligned}
$$

Note that in equations (2.3) and (2.4)

$$
g_{Y M}^{2}=(2 \pi)^{(p-2)} G_{s} \alpha^{\prime \frac{p-3}{2}}, \quad g_{c}=\frac{\kappa_{10}}{2 \pi}=\sqrt{\pi} g_{s}^{2}\left(2 \pi \alpha^{\prime}\right)^{2},
$$

where the open and closed string couplings $G_{s}$ and $g_{s}$ are related by (2.2). The overall normalization constant for the disk amplitudes is given by 31]

$$
C_{D_{2}}=\frac{1}{2 g_{Y M}^{2} \alpha^{\prime 2}} \sqrt{\operatorname{det} G}
$$

and the brane tension is related to the Yang-Mills coupling constant $g_{Y M}$ by

$$
T_{p}=\frac{1}{(2 \pi)^{p} g_{s} \alpha^{\prime \frac{p+1}{2}}}, \quad T_{p} \sqrt{\operatorname{det}(g+B)}=\frac{1}{g_{Y M}^{2}\left(2 \pi \alpha^{\prime}\right)^{2}} \sqrt{\operatorname{det} G}
$$

The Green functions for the worldsheet fermions are ${ }^{5}$

$$
\begin{aligned}
\left\langle\psi^{M}(z) \psi^{N}(w)\right\rangle & =\frac{\alpha^{\prime}}{2} \frac{g^{M N}}{z-w} \\
\left\langle\psi^{M}(z) \tilde{\psi}^{N}(\bar{w})\right\rangle & =\frac{\alpha^{\prime}}{2}\left(\frac{g-B}{g+B} D\right)^{M N} \frac{1}{z-\bar{w}} \\
\left\langle\psi^{M}(z) \Psi^{N}(w)\right\rangle & =\frac{\alpha^{\prime}}{2}\left(\frac{1}{g+B}\right)^{M N} \frac{1}{z-w} \\
\left\langle\tilde{\psi}^{M}(\bar{z}) \Psi^{N}(w)\right\rangle & =\frac{\alpha^{\prime}}{2}\left(\frac{1}{g-B} D\right)^{M N} \frac{1}{\bar{z}-w} \\
\left\langle\Psi^{M}(z) \Psi^{N}(w)\right\rangle & =\frac{\alpha^{\prime}}{2} G^{M N} \frac{1}{z-w} .
\end{aligned}
$$

\footnotetext{
${ }^{5}$ We are being a little sloppy in writing $\frac{g-B}{g+B}$ in 2.18 ) (and subsequent equations) and not specifying $\frac{1}{g}(g-B) \frac{1}{g+B}$ or $\frac{1}{g+B}(g-B) \frac{1}{g}$ because, in fact, they are equal.
} 
The Green functions for $X$ are given by

$$
\begin{gathered}
\left\langle X^{\mu}(z, \bar{z}) X^{\nu}(w, \bar{w})\right\rangle=-\alpha^{\prime}\left[g^{\mu \nu}(\log |z-w|-\log |z-\bar{w}|)+G^{\mu \nu} \log |z-\bar{w}|^{2}\right. \\
\left.+\frac{1}{2 \pi \alpha^{\prime}} \theta^{\mu \nu} \log \frac{z-\bar{w}}{\bar{z}-w}\right] \\
\left\langle X^{i}(z, \bar{z}) X^{j}(w, \bar{w})\right\rangle=-\alpha^{\prime} g^{i j}(\log |z-w|-\log |z-\bar{w}|) .
\end{gathered}
$$

Using the above equations we can work out some basic formulas like

$$
\begin{aligned}
A_{n} & =\left\langle e^{i q \cdot X}(i) \prod_{a=1}^{n} e^{i k_{a} \cdot X}\left(y_{a}\right)\right\rangle \\
& =i 2^{\alpha^{\prime} k^{2}} C_{D_{2}}^{X}(2 \pi)^{d} \delta\left(\sum_{a} k_{a}+q_{\|}\right) \prod_{a<b}\left|\sin \left(\pi \tau_{a b}\right)\right|^{2 \alpha^{\prime} k_{a} \cdot k_{b}} \exp \left[\frac{i}{2}\left(k_{a} \times k_{b}\right)\left(2 \tau_{a b}-\epsilon\left(\tau_{a b}\right)\right)\right]
\end{aligned}
$$

where the $y_{a} \mathrm{~s}$ are on the real axis and $k=\sum_{b} k_{b}$. For later convenience we have expressed the above correlator in terms of $\tau_{i}$ which are defined by $y_{a}=-\cot \left(\pi \tau_{a}\right)$. Note that $0 \leq \tau_{a} \leq 1$ follows from $-\infty<y_{a}<\infty$.

We will also use

$$
B_{n}=a_{M}\left\langle e^{i q \cdot X}(i) i \dot{X}^{M}\left(y_{a}\right) \prod_{b=1}^{n} e^{i k_{b} \cdot X}\left(y_{b}\right)\right\rangle=a_{M} V^{M}\left(y_{a}\right) A_{n}
$$

with

$$
\begin{aligned}
a_{M} V^{M}\left(y_{a}\right)=2 \alpha^{\prime}\left[-(a \cdot k) \frac{y_{a}}{1+y_{a}^{2}}+\frac{i}{2 \pi \alpha^{\prime}}(a \times k) \frac{1}{1+y_{a}^{2}}\right. & +i a_{i} g^{i j} q_{\perp j} \frac{1}{1+y_{a}^{2}} \\
& \left.+\sum_{b=1, b \neq a}^{n}\left(a \cdot k_{b}\right) \frac{1}{y_{a}-y_{b}}\right]
\end{aligned}
$$

where again $k=\sum_{b} k_{b}=-q_{\|}$. In this paper, when not specified otherwise, the dot product is with respect to the open string metric and the cross product denotes contraction using $\theta^{\mu \nu}$, i.e. $a \times k=a_{\mu} \theta^{\mu \nu} k_{\nu}$.

\subsection{One closed and one open}

The amplitude for the interaction of one massless closed string mode and one massless gauge field mode is given by

$$
\begin{aligned}
\mathcal{A} & =\left\langle V_{N S N S}^{-1,-1}(i) V_{O}^{0}(0)\right\rangle \\
& =\frac{2 g_{c}}{\alpha^{\prime}} e_{M N} \operatorname{Tr} a_{P}\left\langle\psi^{M} \tilde{\psi}^{N} e^{i q \cdot X}(i)\left(i \dot{X}^{P}+4 k \cdot \Psi \Psi^{P}\right) e^{i k \cdot X}(0)\right\rangle
\end{aligned}
$$


We have suppressed the ghosts, the relevant contribution from which turns out to be unity. In the following we shall take the polarization of the closed string mode to be along the brane ${ }^{6}$, i.e. $e_{M N}=e_{\mu \nu}$, and we shall always omit the momentum conservation factor $(2 \pi)^{p+1} \delta\left(q_{\|}+\sum_{a} k_{a}\right)$. The amplitude (2.27) can be evaluated using equations (2.24) and (2.25) to yield

$$
\mathcal{A}=\frac{\kappa_{10}}{g_{Y M}^{2}\left(2 \pi \alpha^{\prime}\right)^{2}} \operatorname{Tr}\left[\frac{i}{2} E M-\left(2 \pi \alpha^{\prime}\right) E^{\mu \nu} f_{\mu \nu}\right]
$$

where

$$
E=e_{M N}\left(\frac{g-B}{g+B} D\right)^{M N}, \quad E^{M N}=e_{P Q}\left(\frac{1}{g+B}\right)^{P M}\left(\frac{1}{g-B} D\right)^{Q N}
$$

and

$$
M=\theta^{\mu \nu} a_{\mu} k_{\nu}+\left(2 \pi \alpha^{\prime}\right) \phi_{i} g^{i j} q_{\perp j}, \quad f_{\mu \nu}=i\left(k_{\mu} a_{\nu}-k_{\nu} a_{\mu}\right),
$$

with $\phi_{i} \equiv a_{i}$.

\subsection{One closed and two open}

The amplitude with one massless closed string mode polarized along the brane direction and two noncommutative gauge modes is

$$
\begin{aligned}
\mathcal{A}= & \int_{-\infty}^{\infty} d y\left\langle V_{N S N S}^{-1,-1}(i) V_{O}^{0}(0) V_{O}^{0}(y)\right\rangle \\
= & \frac{2 g_{c}}{\alpha^{\prime}} e_{M N} \operatorname{Tr} a_{1 P} a_{2 Q} \\
& \times \int d y\left\langle\psi^{M} \tilde{\psi}^{N} e^{i q \cdot X}(i)\left(i \dot{X}^{P}+4 k_{1} \cdot \Psi \Psi^{P}\right) e^{i k_{1} \cdot X}(0)\left(i \dot{X}^{Q}+4 k_{2} \cdot \Psi \Psi^{Q}\right) e^{i k_{2} \cdot X}(y)\right\rangle \\
= & \hat{\mathcal{A}}+\tilde{\mathcal{A}}_{1}+\tilde{\mathcal{A}}_{2}
\end{aligned}
$$

For convenience, we have split the amplitude into three parts: $\hat{\mathcal{A}}$ contains the part involving the self-contraction of $\psi$ and $\tilde{\psi}$ in the closed string vertex operator; $\tilde{\mathcal{A}}_{1}$ and $\tilde{\mathcal{A}}_{2}$ contain the remaining terms, which respectively involve four-fermion and six-fermion contractions.

\footnotetext{
${ }^{6} \mathrm{An}$ unfortunate by-product is that we will miss some couplings of the dilaton to the transverse scalar fields and so we will not be able to see explicitly that $\rho_{M}$ of (2.5) drops out in terms that involve scalar fields.
} 
Using (2.24) and (2.25) and integrating over $y$, the final result can be written as

$$
\begin{aligned}
\hat{\mathcal{A}}= & \frac{\kappa_{10}}{2 g_{Y M}^{2}} E J\left[\left(k_{1} \cdot a_{2}\right)\left(k_{2} \cdot a_{1}\right)-\left(a_{1} \cdot a_{2}\right)\left(k_{1} \cdot k_{2}\right)-\frac{1}{\left(2 \pi \alpha^{\prime}\right)^{2}} M_{1} M_{2}\right] \\
& -\frac{\kappa_{10}}{8 \pi \alpha^{\prime} g_{Y M}^{2}} E J \frac{2 \beta}{\alpha^{\prime} t}\left[\left(a_{1} \cdot k_{2}\right) M_{2}-\left(a_{2} \cdot k_{1}\right) M_{1}+\left(k_{1} \times k_{2}\right)\left(a_{1} \cdot a_{2}\right)\right] \\
\tilde{\mathcal{A}}_{1}=- & i \frac{\kappa_{10}}{g_{Y M}^{2}} J E^{\mu \nu}\left[\frac{1}{2 \pi \alpha^{\prime}}\left(f_{1 \mu \nu} M_{2}+f_{2 \mu \nu} M_{1}\right)-\frac{\beta}{\alpha^{\prime} t}\left(f_{1 \mu \nu}\left(a_{2} \cdot k_{1}\right)-f_{2 \mu \nu}\left(a_{1} \cdot k_{2}\right)\right)\right] \\
\tilde{\mathcal{A}}_{2}=- & \frac{\kappa_{10}}{g_{Y M}^{2} J E^{\mu \nu}\left[-f_{1 \mu \rho} f_{2 \nu}^{\rho}+\partial_{\mu} \phi_{1}^{i} \partial_{\nu} \phi_{2 i}+(1 \rightarrow 2)\right.} \\
& \left.-\frac{\beta}{\alpha^{\prime} t}\left(-f_{1 \mu \rho} f_{2 \nu}^{\rho}+\partial_{\mu} \phi_{1}^{i} \partial_{\nu} \phi_{2 i}-(1 \rightarrow 2)\right)\right]
\end{aligned}
$$

where $E$ and $E^{\mu \nu}$ were defined in equations (2.29) and (2.30). $M_{1}$ and $f_{1 \mu \nu}$ generalize (2.30) to

$$
M_{1}=\theta^{\mu \nu} a_{1 \mu} k_{\nu}+\left(2 \pi \alpha^{\prime}\right) \phi_{1 i} g^{i j} q_{\perp j}, \quad f_{1 \mu \nu}=i\left(k_{1 \mu} a_{1 \nu}-k_{1 \nu} a_{1 \mu}\right)
$$

and similarly for $M_{2}, f_{2 \mu \nu}$. Note that the total (open string) momentum $k$ appears in the definition of $M_{1,2}$; this will be important when we relate terms involving $M$ to the Wilson line. We have also introduced

$$
\begin{gathered}
\beta=\frac{k_{1} \times k_{2}}{\pi}, \quad t=-k^{2}=-2 k_{1} \cdot k_{2}, \quad \partial_{\mu} \phi_{1 i}=i k_{1 \mu} \phi_{1 i}, \\
J=\frac{\Gamma\left(1-\alpha^{\prime} t\right)}{\Gamma\left(1-\frac{\alpha^{\prime} t}{2}+\beta\right) \Gamma\left(1-\frac{\alpha^{\prime} t}{2}-\beta\right)}=\frac{\sin \frac{k_{1} \times k_{2}}{2}}{\frac{k_{1} \times k_{2}}{2}}+\mathcal{O}\left(\alpha^{\prime} t\right)+\cdots,
\end{gathered}
$$

In (2.32)-(2.34) the dot product is with respect to the open string metric ${ }^{7}$ and the indices are raised and lowered by the open string metric.

We shall be interested in the leading terms of the amplitude in an $\alpha^{\prime} t$ expansion i.e. we consider the limit $\alpha^{\prime} k_{\mu} G^{\mu \nu} k_{\nu} \ll 1$, while keeping $k_{1 \mu} \theta^{\mu \nu} k_{2 \nu}$ finite. Substituting the leading term in the expansion (2.37) into (2.32)-(2.34), the amplitude separates into finite terms and terms containing poles in $t$. The finite terms are

$$
\begin{aligned}
\hat{\mathcal{A}}_{f} & =\frac{\kappa_{10}}{2 g_{Y M}^{2}} E \frac{\sin \frac{k_{1} \times k_{2}}{2}}{\frac{k_{1} \times k_{2}}{2}}\left[-\frac{1}{\left(2 \pi \alpha^{\prime}\right)^{2}} M_{1} M_{2}+\frac{1}{2} f_{1 \mu \nu} f_{2}^{\mu \nu}+G^{\mu \nu} g^{i j} \partial_{\mu} \phi_{1 i} \partial_{\nu} \phi_{2 j}\right] \\
\tilde{\mathcal{A}}_{1 f} & =i \frac{\kappa_{10}}{g_{Y M}^{2}} \frac{\sin \frac{k_{1} \times k_{2}}{2}}{\frac{k_{1} \times k_{2}}{2}} \frac{1}{2 \pi \alpha^{\prime}} E^{\mu \nu}\left(f_{1 \mu \nu} M_{2}+f_{2 \mu \nu} M_{1}\right)
\end{aligned}
$$

\footnotetext{
${ }^{7}$ In particular $a_{1} \cdot a_{2}=a_{1 \mu} G^{\mu \nu} a_{2 \nu}+a_{1 i} G^{i j} a_{2 j}=a_{1 \mu} G^{\mu \nu} a_{2 \nu}+\phi_{1 i} g^{i j} \phi_{2 j}$.
} 


$$
\begin{aligned}
\tilde{\mathcal{A}}_{2 f}=- & \frac{\kappa_{10}}{g_{Y M}^{2}} \frac{\sin \frac{k_{1} \times k_{2}}{2}}{\frac{k_{1} \times k_{2}}{2}} E^{\mu \nu}\left[-f_{1 \mu \rho} f_{2 \nu}^{\rho}+\partial_{\mu} \phi_{1}^{i} \partial_{\nu} \phi_{2 i}+(1 \rightarrow 2)\right] \\
& +\frac{\kappa_{10}}{\pi \alpha^{\prime} g_{Y M}^{2}} \sin \frac{k_{1} \times k_{2}}{2} E^{\mu \nu}\left(a_{1 \mu} a_{2 \nu}-a_{2 \mu} a_{1 \nu}\right),
\end{aligned}
$$

and the terms with a pole are

$$
\begin{aligned}
& \hat{\mathcal{A}}_{p}=-\frac{\kappa_{10}}{2 \pi^{2} \alpha^{\prime} g_{Y M}^{2}} E \frac{\sin \frac{k_{1} \times k_{2}}{2}}{\alpha^{\prime} t}\left[\left(a_{1} \cdot k_{2}\right) M_{2}-\left(a_{2} \cdot k_{1}\right) M_{1}+\left(k_{1} \times k_{2}\right)\left(a_{1} \cdot a_{2}\right)\right] \\
& \tilde{\mathcal{A}}_{1 p}=\frac{2 i}{\pi} \frac{\kappa_{10}}{g_{Y M}^{2}} \frac{\sin \frac{k_{1} \times k_{2}}{2}}{\alpha^{\prime} t} E^{\mu \nu}\left[f_{1 \mu \nu}\left(a_{2} \cdot k_{1}\right)-f_{2 \mu \nu}\left(a_{1} \cdot k_{2}\right)\right] \\
& \tilde{\mathcal{A}}_{2 p}=\frac{2}{\pi} \frac{\kappa_{10}}{g_{Y M}^{2}} \frac{\sin \frac{k_{1} \times k_{2}}{2}}{\alpha^{\prime} t} E^{\mu \nu}\left[-\left(f_{1 \mu \rho} f_{2 \nu}^{\rho}\right)_{p}+\partial_{\mu} \phi_{1}^{i} \partial_{\nu} \phi_{2 i}-(1 \rightarrow 2)\right]
\end{aligned}
$$

where

$$
\left[f_{1 \mu \lambda} f_{2 \nu}^{\lambda}\right]_{p}=-\left[\left(a_{2} \cdot k_{1}\right) a_{1 \mu} k_{2 \nu}+\left(a_{1} \cdot k_{2}\right) a_{2 \nu} k_{1 \mu}-\left(a_{1} \cdot a_{2}\right) k_{1 \mu} k_{2 \nu}\right]
$$

Note that the fourth term in $f_{1 \mu \lambda} f_{2}^{\lambda}$ contributes to the finite term. The pole terms are all proportional to $\sin \frac{k_{1} \times k_{2}}{2}$ and can be reproduced from exchanging massless gauge bosons (or scalar fields) using the vertex (2.28) and cubic vertices of gauge bosons, as was pointed out in [21].

The finite terms in $(2.38)-(2.40)$ are all proportional to $\frac{\sin \frac{k_{1} \times k_{2}}{2}}{\frac{k_{1} \times k_{2}}{2}}$, except for the last term in (2.40) which is proportional to $\sin \frac{k_{1} \times k_{2}}{2}$. The factor $\frac{\sin \frac{k_{1} \times k_{2}}{2}}{\frac{k_{1} \times k_{2}}{2}}$ gives rise to the generalized star-product $*_{2}$ between two Yang-Mills fields [21], which in momentum space is

$$
f\left(k_{1}\right) *_{2} g\left(k_{2}\right)=f\left(k_{1}\right) \frac{\sin \frac{k_{1} \times k_{2}}{2}}{\frac{k_{1} \times k_{2}}{2}} g\left(k_{2}\right)
$$

The last term of (2.40) combines with the second term in (2.28) to give the noncommutative field strength

$$
F_{\mu \nu}=\partial_{\mu} A_{\nu}-\partial_{\nu} A_{\mu}-i A_{\mu} * A_{\nu}+i A_{\nu} * A_{\mu}
$$

which in momentum space becomes

$$
F_{\mu \nu}=i\left(k_{\mu} A_{\nu}-k_{\nu} A_{\mu}\right)-2 \sin \frac{k_{1} \times k_{2}}{2} A_{\mu}\left(k_{1}\right) A_{\nu}\left(k_{2}\right)
$$




\section{Supergravity Couplings of Noncommutative D-branes}

From equations (2.28) and (2.38)-(2.40) we are ready to write down the effective couplings of the massless NSNS closed string modes to the noncommutative D-brane up to terms quadratic in Yang-Mills fields. Since $M$ defined in (2.30) and (2.35) involve the total momentum of the open string modes it is more convenient to write down the couplings in momentum space ${ }^{8}$

$$
\begin{gathered}
S=\frac{\kappa_{10}}{g_{Y M}^{2}\left(2 \pi \alpha^{\prime}\right)^{2}} \int \frac{d^{10} q}{(2 \pi)^{10}} \frac{d^{p+1} k_{1}}{(2 \pi)^{p+1}} \frac{d^{p+1} k_{2}}{(2 \pi)^{p+1}} \sqrt{\operatorname{det} G}(2 \pi)^{p+1} \delta^{(p+1)}\left(q_{\|}+k_{1}+k_{2}\right) \\
\times\left\{\frac{1}{2} E(q)\left[1+i M\left(k_{1}\right)-\frac{1}{2} M\left(k_{1}\right) *_{2} M\left(k_{2}\right)+\frac{1}{4} \mathcal{F}_{\mu \nu}\left(k_{1}\right) *_{2} \mathcal{F}^{\mu \nu}\left(k_{2}\right)+\frac{1}{2} D_{\mu} X_{i}\left(k_{1}\right) *_{2} D^{\mu} X^{i}\left(k_{2}\right)\right]\right. \\
\left.+\frac{1}{2} E^{\mu \nu}(q)\left[-\mathcal{F}_{\mu \nu}\left(k_{1}\right)-i \mathcal{F}_{\mu \nu}\left(k_{1}\right) *_{2} M\left(k_{2}\right)+\mathcal{F}_{\mu \rho}\left(k_{1}\right) *_{2} \mathcal{F}^{\rho}{ }_{\nu}\left(k_{2}\right)-D_{\mu} X^{i}\left(k_{1}\right) *_{2} D_{\nu} X_{i}\left(k_{2}\right)\right]\right\}
\end{gathered}
$$

where $\mathcal{F}=2 \pi \alpha^{\prime} F$ is the noncommutative field strength (2.46), $X_{i}=2 \pi \alpha^{\prime} \phi_{i}$ and $D_{\mu} X_{i}=\partial_{\mu} X_{i}-i\left[A_{\mu}, X_{i}\right]_{*}$. Note that we have promoted the perturbation $a_{M}$ to the full gauge field $A_{M} . \mathcal{F}$ and $D X$ should be understood as their Fourier transforms, as in (2.47). For terms linear in Yang-Mills fields it is understood that $k_{2}$ should be set to zero and not integrated, and for the tadpole term, it is understood that $k_{1}=k_{2}=0$. Except for those in $E$ and $E^{\mu \nu}$ the indices are always raised and lowered using the open string metric (recall $G_{i j}=g_{i j}$ ). The $*_{2}$-product was defined in (2.45) and

$$
M\left(k_{i}\right)=\theta^{\mu \nu} q_{\mu} A_{\nu}\left(k_{i}\right)+q_{\perp} \cdot X\left(k_{i}\right) .
$$

For the graviton polarized along the brane

$$
E=h_{\mu \nu}\left(\frac{g-B}{g+B}\right)^{\mu \nu}, \quad E^{\mu \nu}=h_{\lambda \rho}\left(\frac{1}{g+B}\right)^{\lambda \mu}\left(\frac{1}{g-B}\right)^{\rho \nu} .
$$

with $h_{\mu \nu}$ symmetric, traceless in terms of closed string metric, i.e. $g^{\mu \nu} h_{\mu \nu}=0$. A similar expression applies to the NSNS anti-symmetric tensor $b_{\mu \nu}$. Note $E$ and $E^{\mu \nu}$ in (3.3) satisfy the relation

$$
E=G_{\mu \nu} E^{\nu \mu}
$$

For the dilaton

$$
E=\varphi\left(g_{\mu \nu} G^{\mu \nu}-4\right), \quad E^{\mu \nu}=\frac{1}{2} \varphi g_{\lambda \rho}\left(\frac{1}{g+B}\right)^{\lambda \mu}\left(\frac{1}{g-B}\right)^{\rho \nu}
$$

\footnotetext{
${ }^{8}$ We have included a tadpole term which can be easily obtained, for example, by cutting open the annulus diagram.
} 
It can be checked that the effective action (3.1) is not gauge invariant under the noncommutative gauge transformations

$$
\begin{aligned}
& \delta_{\lambda} A_{\mu}=\partial_{\mu} \lambda+i \lambda * A_{\mu}-i A_{\mu} * \lambda, \\
& \delta_{\lambda} X_{i}=i \lambda * X_{i}-i X_{i} * \lambda .
\end{aligned}
$$

This is almost the same situation encountered in the one-loop effective action of noncommutative super-Yang-Mills theory [8, 9], which is related to the disk amplitudes considered here by factorization. The reason is by now well understood; the non-gaugeinvariance of higher order terms in the action will conspire to cancel those from (3.1) to make the whole effective action gauge invariant [7, 6, 32]. Using the result of [6] regarding the relation between the $*_{n}$ products and an open Wilson line, the resolution is to attach each $\mathcal{F}$ and $X$ in (3.1) to a straight Wilson line and integrate over the insertion positions with respect to the path ordering. The presence of the Wilson line can already been seen from the nature of the $M$-dependence in (3.1). Thus we obtain the following gauge invariant completion of the above action (3.1)

$$
S=\frac{\kappa_{10}}{g_{Y M}^{2}\left(2 \pi \alpha^{\prime}\right)^{2}} \int \frac{d^{10} q}{(2 \pi)^{10}} \sqrt{\operatorname{det} G} \frac{1}{2} E^{\mu \nu}(q) T_{\mu \nu}(-q)
$$

where

$$
\begin{aligned}
T_{\mu \nu}(-q) & =\operatorname{Tr} \int d^{p+1} x L_{*}\left(W(x, C) T_{\mu \nu}(x)\right) * e^{i q_{\rho} x^{\rho}} \\
T_{\mu \nu}(x) & =G_{\mu \nu}\left(1+\frac{1}{4} \mathcal{F}_{\lambda \rho} \mathcal{F}^{\lambda \rho}+\frac{1}{2} D_{\lambda} X_{i} D^{\lambda} X^{i}\right)-\mathcal{F}_{\mu \nu}+\mathcal{F}_{\mu \rho} \mathcal{F}^{\rho}{ }_{\nu}-D_{\mu} X^{i} D_{\nu} X_{i}
\end{aligned}
$$

and $\left(\xi^{\nu}(\tau)=\theta^{\mu \nu} q_{\mu} \tau\right)$

$$
W(x, C)=P_{*} \exp \left[i \int_{0}^{1} d \tau\left(q_{\mu} \theta^{\mu \nu} A_{\nu}(x+\xi(\tau))+q_{\perp i} X^{i}(x+\xi(\tau))\right)\right] .
$$

Clearly, (3.7c) leads to the expansion in $M$ seen in (3.1). In (3.7b) we have used a short hand notation $L_{*}$ to denote the procedure of integrating each $\mathcal{F}$ or $X$ in $T_{\mu \nu}$ along the Wilson line via path ordering. ${ }^{9}$

\footnotetext{
${ }^{9}$ For example,

$\int d^{p+1} x L_{*}\left[W(x, C) F_{\mu \rho}(x) F_{\nu}^{\rho}(x)\right] * e^{i q_{\mu} x^{\mu}}$ $=\int d^{p+1} x \int_{0}^{1} d \tau_{1} \int_{0}^{1} d \tau_{2} P_{*}\left[W(x, C) F_{\mu \rho}\left(x+\xi\left(\tau_{1}\right)\right) F^{\rho}{ }_{\nu}\left(x+\xi\left(\tau_{2}\right)\right)\right] * e^{i q \cdot x}$.
} 
In general,

$$
\begin{aligned}
& \int d^{p+1} x L_{*}\left[W(x, C) \prod_{a=1}^{n} \mathcal{O}_{a}(x)\right] * e^{i q \cdot x} \\
& \equiv \int d^{p+1} x\left(\prod_{a=1}^{n} \int_{0}^{1} d \tau_{a}\right) P_{*}\left[W(x, C) \prod_{a=1}^{n} \mathcal{O}_{a}\left(x+\xi\left(\tau_{a}\right)\right)\right] * e^{i q \cdot x} \\
& =\sum_{m=0}^{\infty} \frac{i^{m}}{m !}\left(\prod_{i=a}^{n+m} \int \frac{d^{p+1} k_{a}}{(2 \pi)^{p+1}}\right) Q_{m}\left(k_{1}, \cdots, k_{n+m}\right)
\end{aligned}
$$

where in the third line we have expanded the Wilson line in terms of the power series of the gauge fields with $\left(\mathcal{O}_{i}(k)\right.$ are the Fourier transforms of $\left.\mathcal{O}_{i}(x)\right)$

$Q_{m}=(2 \pi)^{p+1} \delta^{(p+1)}\left(q+\sum_{i=1}^{m+n} k_{i}\right) \mathcal{O}_{1}\left(k_{1}\right) \cdots \mathcal{O}_{n}\left(k_{n}\right) M\left(k_{n+1}\right) \cdots M\left(k_{n+m}\right) J_{n+m}\left(k_{1}, \cdots, k_{n+m}\right)$

and

$$
J_{n}\left(k_{1}, \cdots, k_{n}\right)=\int_{0}^{1} d \tau_{1} \cdots \int_{0}^{1} d \tau_{n} \exp \left[\frac{i}{2} \sum_{a<b}^{n}\left(k_{a} \times k_{b}\right)\left(2 \tau_{a b}-\epsilon\left(\tau_{a b}\right)\right)\right] .
$$

It is important to note that all the entries in (3.9) are completely symmetric under the ordering due to symmetric properties of the $J_{m+n}$. For simplicity, we have written equations (3.10)-(3.12) as if the gauge group were abelian. With Chan-Paton factors, we should include a path ordering with respect to the $\tau_{i}$ s when multiplying the adjointvalued objects; this orders the operators according to their location on the Wilson line. This prescription clearly maintains the full symmetry of $J_{m+n}$.

In the next section we shall show that higher order terms in (3.7) can be systematically extracted from the amplitudes of one closed string with arbitrary number of open string modes. In particular, the structure of the amplitude is such that one obtains a fully symmetrized action, though only in the low energy limit.

The effective action (3.7) indicates that when we turn on a constant $B$-field, the D-brane becomes a source for the dilaton (even for a D3-brane) and the NSNS $B$-field, in addition to the graviton. This is expected as we know that the supergravity solution for D3-branes with a $B$-field involves nontrivial dilaton and $B$-field background [33, 34]. Since a constant $B$-field does not break supersymmetry, there will still be no net force between parallel D-branes. Thus the exchange amplitude due to these additional couplings have to cancel with the new couplings in the R-R sector or among themselves. 
Similarly there are new couplings at the linear and quadratic level, and their contributions to $F^{2}$ and $F^{3}$ interactions between the branes should cancel one another or with those in RR-sector since, as shown in [8], there is no one-loop correction to $F^{2}$ and $F^{3}$ terms in $\mathcal{N}=4$ noncommutative super-Yang-Mills theory. In [6], minimal couplings which reproduce the one-loop $F^{4}$ terms were worked out and it was found for example that the graviton couples to noncommutative gauge field modes in a form

$$
h_{\lambda \rho}(q) g^{\lambda \mu} G^{\rho \nu} \int d^{p+1} x L_{*}\left[W(x, C)\left(F_{\mu \sigma} F_{\nu}{ }^{\sigma}-\frac{1}{4} G_{\mu \nu} F_{\sigma \alpha} F^{\sigma \alpha}\right)\right] * e^{i q_{\rho} x^{\rho}} .
$$

The quadratic in field strength coupling to the graviton that we found above is

$$
h_{\lambda \rho}(q)\left(\frac{1}{g+B}\right)^{\lambda \mu}\left(\frac{1}{g-B}\right)^{\rho \nu} \int d^{p+1} x L_{*}\left[W(x, C)\left(F_{\mu \sigma} F_{\nu}{ }^{\sigma}-\frac{1}{4} G_{\mu \nu} F_{\sigma \alpha} F^{\sigma \alpha}\right)\right] * e^{i q_{\rho} x^{\rho}}
$$

The general structures of (3.11) and (3.12) agree very well except for the index structure of the graviton. This is to be expected since (3.11) was inferred indirectly to reproduce the one-loop $F^{4}$ terms and this procedure does not determine the Lorentz structure uniquely.

To gain some intuition about the physical meaning of (3.7) let us look at some special cases.

\section{$3.1 B=0$}

In this case $E^{\mu \nu}$ is the standard supergravity perturbation and equation (3.7b) becomes

$$
T_{\mu \nu}(-q)=\int d^{p+1} x e^{i q_{\perp i} X^{i}} e^{i q_{\| \rho} x^{\rho}} T_{\mu \nu}(x)
$$

and thus equation (3.7a) becomes

$$
\begin{aligned}
S & =\frac{\kappa_{10}}{g_{Y M}^{2}\left(2 \pi \alpha^{\prime}\right)^{2}} \int \frac{d^{10} q}{(2 \pi)^{10}} \sqrt{\operatorname{det} G} \frac{1}{2} E^{\mu \nu}(q)\left[\int d^{p+1} x e^{i q_{\perp i} X^{i}} e^{i q_{\| \rho} x^{\rho}} T_{\mu \nu}(x)\right] \\
& =\frac{\kappa_{10}}{g_{Y M}^{2}\left(2 \pi \alpha^{\prime}\right)^{2}} \int d^{p+1} x \sqrt{\operatorname{det} G} \frac{1}{2} E^{\mu \nu}(X(x), x) T_{\mu \nu}(x)
\end{aligned}
$$

where in the above we have used

$$
E^{\mu \nu}(X(x), x)=\int \frac{d^{10} q}{(2 \pi)^{10}} E^{\mu \nu}(q) e^{i q_{\perp i} X^{i}} e^{i q_{\| \rho} x^{\rho}}
$$

The above result indicated that the external supergravity fields should be considered as a functional of the transverse scalar fields. 
In the nonabelian case, the $L_{*}$ in $(3.7 \mathrm{~b})$ reduces to a symmetrized trace and the action becomes:

$$
S=\frac{\kappa_{10}}{g_{Y M}^{2}\left(2 \pi \alpha^{\prime}\right)^{2}} \int d^{p+1} x \sqrt{\operatorname{det} G} \frac{1}{2} \operatorname{STr}\left[E^{\mu \nu}\left(X_{i}(x), x\right) T_{\mu \nu}(x)\right]
$$

Note that the $X_{i}(x)$ are now nonabelian matrices and the symmetrized trace is to be understood as first doing the Fourier transform as in (3.15) and then treat the exponential as a power series.

\subsection{Relation with Matrix Theory}

In this section we shall consider the Euclidean version [35] of Matrix Theory [36]. Noncommutative Yang-Mills theory is obtained naturally from the $U(\infty)$ limit of Matrix Theory [13, 14, 11, 15]. This is closely related to the construction of D $p$-Branes in Matrix Theory (see e.g. [11, 12, 14]). In terms of Matrix Theory variables $X^{M}, \mathrm{D} p$-branes can be obtained as a classical solution

$$
\left[x^{\mu}, x^{\nu}\right]=i \theta^{\mu \nu}, \quad \mu, \nu=0, \cdots, p
$$

and the noncommutative Yang-Mills theory arises by considering the dynamics of the fluctuations around the classical solution

$$
X^{\mu}=x^{\mu}+\theta^{\mu \nu} A_{\nu}
$$

and

$$
\left[X^{\mu}, X^{\nu}\right]=-i\left[\theta\left(F-\theta^{-1}\right) \theta\right]^{\mu \nu}
$$

As pointed out in [15], the noncommutative Yang-Mills theory obtained this way corresponds to the choice of $\Phi=-B$ description of the open string dynamics [5]. In this description, the open string parameters given by

$$
\begin{aligned}
\theta & =\frac{1}{B} \\
G^{-1} & =-\frac{1}{B} g \frac{1}{B} \\
G_{s} & =g_{s} \sqrt{\operatorname{det} B g^{-1}}
\end{aligned}
$$

On the other hand the off-shell action (3.7a)-(3.7d), which was extrapolated from the on-shell string amplitudes, corresponds to the choice $\Phi=0$. The two descriptions are in general related by field redefinitions [5]. 
In the large $B$-field limit, the mixed Neumann and Dirichlet boundary conditions (2.7) become effectively Dirichlet conditions and thus we expect that our results (3.7a)-(3.7d) can be expressed naturally in terms of Matrix Theory variables $X$. Indeed it can be checked that equations (2.1) and (2.2) reduce to (3.20)-(3.22) in this limit. We note that the open Wilson line (3.7d) can be written in Matrix Theory variables (3.18) as [37]

$$
W(x, C)=\exp (i q \cdot X)=\exp \left(i q_{\mu} X^{\mu}+i q_{i} X^{i}\right)
$$

After some algebraic manipulations it can be shown that in the large $B$-field limit, the action (3.7a)-(3.7g) can be rewritten (e.g. for the graviton) as

$$
S=\frac{\kappa_{10}}{(2 \pi)^{\frac{p-1}{2}} g_{s} \alpha^{\prime\left(\frac{p+1}{2}\right)}} \int \frac{d^{10} q}{(2 \pi)^{10}} \sqrt{\operatorname{det} \theta} h_{\mu \nu}(q) \operatorname{S} \operatorname{Tr}\left[e^{i q \cdot X}\left[X^{\mu}, X^{M}\right] g_{M N}\left[X^{N}, X^{\nu}\right]\right]
$$

where we have used (3.19). In the above "Tr" should be understood to be defined in the Hilbert space given by (3.17), i.e.

$$
\sqrt{\operatorname{det} \theta} \operatorname{Tr}=\int \frac{d^{p+1} x}{(2 \pi)^{\frac{p+1}{2}}} \operatorname{tr}
$$

where here "tr" denotes the group trace, and the symmetrized trace "STr" should be understood as a formal way of writing the $L_{*}$ prescription defined below (3.70).

\section{The structure of higher-point amplitudes and the open Wil- son line}

In this section we will show how, by considering disk amplitudes with one massless closed string and an arbitrary number of massless open string modes, one finds the full open Wilson line in the effective action (3.7). We will not attempt to evaluate the general amplitudes and shall be only interested in the parts of the amplitudes relevant for the higher order terms in (3.7). As we have seen the last section, they should arise as finite terms of the amplitudes in the limit $\alpha^{\prime} k_{a} \cdot k_{b} \rightarrow 0 .{ }^{10}$ For the convenience of the discussion we shall not write explicitly the numerical prefactors of the amplitudes.

\footnotetext{
${ }^{10}$ We will ignore terms with a pole in $k_{a} \cdot k_{b}$ as they arise from exchanging massless gauge bosons (or scalars) using lower order vertices.
} 
The general disk amplitude is

$$
\begin{aligned}
\mathcal{A}_{m} & =\left[\prod_{a=2}^{m} \int_{-\infty}^{\infty} d y_{a}\right]\left\langle V_{N S N S}^{-1,-1}(i) \prod_{a=1}^{m} V_{O}^{0}\left(\xi_{a}, k_{a} ; y_{a}\right)\right\rangle \\
& =e_{M N} \int d y\left\langle\psi^{M} \tilde{\psi}^{N} e^{i q \cdot X}(i) \prod_{a=1}^{m} a_{a M_{a}}\left(i \dot{X}^{M_{a}}+4 k_{a} \cdot \Psi \Psi^{M_{a}}\right) e^{i k_{1} \cdot X}\left(y_{a}\right)\right\rangle .
\end{aligned}
$$

We may take $y_{1}=0$ using the $S L(2, \mathbb{R})$ invariance of the disk, and in the second line we have used a shorthand for all the $y_{a}$-integrations.

There are two important features of the correlators inside the integrals which will be relevant for us. There is a nontrivial phase factor

$$
\prod_{a<b} \exp \left[\frac{i}{2}\left(k_{a} \times k_{b}\right)\left(2 \tau_{a b}-\epsilon\left(\tau_{a b}\right)\right)\right]
$$

as was given in (2.24). (Note that this just gives unity when $B=0$.) The $\epsilon\left(\tau_{a b}\right)$ term is probably quite familiar by now, from [5] and much subsequent work. It arises from bosonic contractions between open string vertex operators. Were it not for the linear term, which arises from contractions between the open strings and the closed string, the phase factor would give rise to the $*$-product. ${ }^{11}$ Equation (4.2) is precisely the same phase factor that appears in the expansion of the open Wilson lines (3.10).

Another general feature is that for every $i \dot{X}^{M}\left(y_{a}\right)$ insertion from an open string vertex operator, there is a term of the form

$$
i\left[\left(q \times a_{a}\right)+\left(2 \pi \alpha^{\prime}\right) \phi_{a} \cdot q_{\perp}\right] \frac{1}{\pi\left(1+y_{a}^{2}\right)},
$$

coming from the contraction with $e^{i q \cdot X}$ in the closed string vertex operator, as was already given in (2.26). This, of course, is precisely the $i M_{a}$ of (3.2).

Naïvely, the Wilson lines now can be seen to arise as follows. Suppose we are considering what is an interaction between $n$ open strings and a closed string. For each additional open string that we add, one of the contributions is an additional factor of $i M_{a}$. Because this comes from the $i \dot{X}^{M}\left(y_{a}\right)$ in the vertex operator, the rest of the amplitude is almost unchanged from that without the additional open string; the only other change is in the phase factor (4.2) from the additional $e^{i k X}$. But this is precisely the open Wilson line expansion (3.8)-(3.10). In practice the story is more complicated as there are additional functions and singularities of of $y_{a}$ in the integrand.

\footnotetext{
${ }^{11}$ The linear term vanishes when there is no momentum flow between the closed and open strings. The phase factor also appeared in one-loop diagrams [8]; this is more than just a coincidence as the one-loop diagrams must factorize into tree diagrams.
} 
As the simplest example let us look at the (tadpole) term

$$
E(q) \int d^{p+1} x W(x, C) * e^{i q_{\mu} x^{\mu}}
$$

in (3.7). From what was outlined just after equation (4.3), the relevant terms are the products of $i M$ coming from the contraction of $i \dot{X}$ with the $e^{i q \cdot X}$ part of the closed string vertex operator. Thus the relevant terms are

$$
\begin{aligned}
\int_{-\infty}^{\infty} d y A_{n} \prod_{b=1}^{m} \frac{i}{\pi} M\left(k_{b}\right) \frac{1}{1+y_{b}^{2}} & \\
=(2 \pi)^{d} \delta\left(\sum_{a} k_{a}+q_{\|}\right) \int_{-\infty}^{\infty} d y\left\{\prod _ { a < b } \left[\left|\sin \left(\pi \tau_{a b}\right)\right|^{2 \alpha^{\prime} k_{a} \cdot k_{b}}\right.\right. & \left.\exp \left[\frac{i}{2}\left(k_{a} \times k_{b}\right)\left(2 \tau_{a b}-\epsilon\left(\tau_{a b}\right)\right)\right]\right] \\
& \left.\times \prod_{b=1}^{m} i M\left(k_{b}\right) \frac{1}{\pi\left(1+y_{b}^{2}\right)}\right\}
\end{aligned}
$$

where $y_{a}=-\cot \pi \tau_{a}$ and in the first line $A_{n}$ is given by (2.24). Since the integrand (4.5) is regular for all values of $y_{a}$ we can take the limit $\alpha^{\prime} k_{a} \cdot k_{b} \rightarrow 0$ before integrating over the vertex operator positions ${ }^{12}$. Using $\frac{d y}{d \tau}=\pi\left(1+y^{2}\right)$ to change the integration variables to $\tau_{a}$ we find

$$
(2 \pi)^{p+1} \delta\left(\sum_{a} k_{a}+q_{\|}\right) \prod_{b=1}^{m} i M\left(k_{b}\right)\left(\prod_{a=2}^{m} \int_{0}^{1} d \tau_{a}\right) \prod_{a<b=1}^{m} \exp \left[\frac{i}{2}\left(k_{a} \times k_{b}\right)\left(2 \tau_{a b}-\epsilon\left(\tau_{a b}\right)\right)\right] .
$$

This is precisely the $m$ th-order term in the expansion of a Wilson line (3.8)-(3.10) with no operator insertion ${ }^{13}$. The factor of $\frac{1}{m !}$ also arises correctly from the symmetry factor when translating the amplitude into the effective action.

As a more complicated example let us look at the term

$$
E(q) \int d^{p+1} x\left[W(x, C) F_{\mu \nu} F^{\mu \nu}\right] * e^{i q_{\rho} x^{\rho}}
$$

in (3.7), which has an expansion, using (3.8)-(3.10), of

$$
\sum_{m=0}^{\infty} \frac{1}{m !}\left(\prod_{a=1}^{m+2} \int \frac{d^{p+1} k_{a}}{(2 \pi)^{p+1}}\right) F_{\mu \nu}\left(k_{1}\right) F^{\mu \nu}\left(k_{2}\right)\left(\prod_{b=3}^{m+2} i M\left(k_{b}\right)\right) J_{m+2}\left(k_{1}, \cdots, k_{m+2}\right)
$$

\footnotetext{
${ }^{12}$ Although we are interested in the $\alpha^{\prime} k_{a} \cdot k_{b} \rightarrow 0$ limit, we cannot generally take this limit until after integrating, because the integrals are typically defined only by analytic continuation from outside the kinematic region of interest. We can take $\alpha^{\prime} k_{a} \cdot k_{b} \rightarrow 0$ inside the integrals only when the integrand is regular throughout the limit.

${ }^{13}$ Recall that the $S L(2, \mathbb{R})$-invariance was used to fix $y_{1}=0$ or $\tau_{1}=\frac{1}{2}$.
} 
where we have omitted the factor $(2 \pi)^{p+1} \delta\left(q+\sum_{a=1}^{m+2} k_{a}\right)$ so as not to make the formula too long. To reproduce the $m$ th order term in (4.8) we shall look at the following amplitude

$$
\int_{-\infty}^{\infty} d y_{2} \prod_{a=3}^{m+2} d y_{a}\left\langle V_{N S N S}^{-1,-1}(i) V_{O}^{0}\left(0 ; k_{1}, a_{1}\right) V_{O}^{0}\left(y_{2} ; k_{2}, a_{2}\right) \prod_{a=3}^{m} V_{O}^{0}\left(y_{a} ; k_{a}, a_{a}\right)\right\rangle
$$

where we have separated two open string vertex operators with the rest. For vertex operators $a=3, \cdots, m+2$ we again take only the factors $i M_{a}$ while the first two are to be contracted with the closed string vertex operator in a way that gives rise to the factor $F_{\mu \nu}\left(k_{1}\right) F^{\mu \nu}\left(k_{2}\right)=2\left(k_{1} \cdot a_{2}\right)\left(k_{2} \cdot a_{1}\right)-2\left(k_{1} \cdot k_{2}\right)\left(a_{1} \cdot a_{2}\right)$. With this in mind it is easy to extract terms of the relevant kinematic structure from the amplitude (4.9)

$$
\begin{array}{r}
E(q) \int_{-\infty}^{\infty}\left(d y_{2} \prod_{i=a}^{m+2} d y_{a}\right) A_{m+2}\left[2 \alpha^{\prime}\left(a_{1} \cdot k_{2}\right)\left(a_{2} \cdot k_{1}\right) \frac{1}{1+y_{2}^{2}}+\frac{1}{y_{2}^{2}}\left(1+\alpha^{\prime} t\right)\left(a_{1} \cdot a_{2}\right)\right] \\
\times \prod_{a=3}^{m+2} \frac{i M_{a}}{\pi\left(1+y_{a}^{2}\right)}
\end{array}
$$

where $t=-2 k_{1} \cdot k_{2}$ and $A_{m+2}$ is again given by (2.24). The first term in the square bracket is regular as we take $\alpha^{\prime} k_{a} \cdot k_{b} \rightarrow 0$ and after changing the integration coordinates to $\tau_{a}$ we find,

$$
2 \pi \alpha^{\prime} E(q)\left(k_{1} \cdot a_{2}\right)\left(k_{2} \cdot a_{1}\right)\left(\prod_{j=3}^{m+2} i M_{j}\right) J_{m+2}\left(k_{1}, \cdots, k_{m+2}\right)
$$

The second term has a double pole in $y_{2}$ as we take $\alpha^{\prime} k_{1} \cdot k_{2}$ to zero. Thus we can not take the $\alpha^{\prime} k_{1} \cdot k_{2} \rightarrow 0$ naïvely in the integrand and have to be more careful with the integral over $y_{2}$. Taking all other $\alpha^{\prime} k_{i} \cdot k_{j}$ to zero except $\alpha^{\prime} k_{1} \cdot k_{2}$ and changing the coordinates to $\tau_{i}$ we get $^{14}$

$$
\begin{aligned}
E(q) \pi\left(a_{1} \cdot a_{2}\right) \prod_{a=3}^{m+2} i M_{a}\left(1+\alpha^{\prime} t\right)\left(\int_{0}^{1} d \tau_{2}\right. & \left.\prod_{a=3}^{m+2} d \tau_{a}\right)\left|\cos \pi \tau_{2}\right|^{-\alpha^{\prime} t-2} \\
& \times \prod_{a<b=1}^{m+2} \exp \left[\frac{i}{2}\left(k_{a} \times k_{b}\right)\left(2 \tau_{a b}-\epsilon\left(\tau_{a b}\right)\right)\right] .
\end{aligned}
$$

Note an identity

$$
\left(1+\alpha^{\prime} t\right)\left|\cos \pi \tau_{2}\right|^{-\alpha^{\prime} t-2}=\alpha^{\prime} t\left|\cos \pi \tau_{2}\right|^{-\alpha^{\prime} t}+\frac{1}{\pi^{2} \alpha^{\prime} t} \partial_{\tau_{2}}^{2}\left|\cos \pi \tau_{2}\right|^{-\alpha^{\prime} t}
$$

\footnotetext{
${ }^{14}$ Recall that $y_{1}=0$ and so $\tau_{1}=\frac{1}{2}$.
} 
Now both terms on the right hand side are regular in $\tau_{2}$ as we take $\alpha^{\prime} k_{1} \cdot k_{2} \rightarrow 0$, where the second term should be evaluated using integration by parts. Since integrating by parts in the second term does not yield anything to cancel the $1 /\left(k_{1} \cdot k_{2}\right)$ factor, it contributes a pole term in the final amplitude and is not of interest to us. Thus in the low energy limit (4.12) becomes

$$
-2 \alpha^{\prime} E\left(a_{1} \cdot a_{2}\right)\left(k_{1} \cdot k_{2}\right) \prod_{a=3}^{m+2} i M_{a}\left(\int_{0}^{1} \prod_{a=2}^{m+2} d \tau_{a}\right) \prod_{a<b=1}^{m+2} \exp \left[\frac{i}{2}\left(k_{a} \times k_{b}\right)\left(2 \tau_{a b}-\epsilon\left(\tau_{a b}\right)\right)\right] .
$$

Combining (4.11) and (4.14) we find precisely (4.8) including the symmetric factor. We have again suppressed the Chan-Paton factors, but they can again be easily inserted. In particular, when $B=0$, only the second term in (4.3) survives, which leads to the fully symmetrized trace over the Chan-Paton factors. We also note that if at the beginning we had taken the large $B$ limit of section 3.2, then the $F^{2}$ term we have just finished considering would not have appeared.

Similarly we can recover all higher order terms in (3.7), by specifying a contraction as in the corresponding term in (3.1) by extracting factors of $i M_{a}$ from other contractions.

\section{Discussion and Conclusions}

In this paper we have investigated the supergravity couplings of the noncommutative D-branes. The general picture that emerges agrees very well with the proposal in [6] and with the expectations from the Matrix Model[10]: supergravity fields couple to an open Wilson line and additional operator insertions have to be integrated along the Wilson line. When $B=0$, this prescription reduces to the "symmetrized trace" prescription for multiple D-branes interacting with background supergravity fields, and the supergravity fields are seen to be functionals of the nonabelian scalar fields on the branes.

When $B \neq 0$, the graviton, antisymmetric tensor and the dilaton all couple to Yang-Mills fields at tadpole, linear and quadratic orders. Most of these couplings disappear when $B=0$. Then, for example, the dilaton only couples to the D3-brane at quadratic level. Since, from the worldsheet point of view turning on a constant $B$-field simply corresponds to a relative Lorentz rotation between the left and right moving sector, we may consider the couplings at $B \neq 0$ as certain kind of rotation of the original $B=0$ couplings. Indications of this can be seen from the Lorentz index structure of (3.3)-(3.5) (see also equation (5.4) below). This point of view may be 
helpful in understanding the mixings in the perturbations of supergravity background in the noncommutative version of $\mathrm{AdS} / \mathrm{CFT}$ [33, 34].

Finally we comment on the relation between our results and the Born-Infeld action. The effective action (3.7a)-(3.7c) can be obtained from the ordinary Born-Infeld action by the following procedure:

1. Take the Born-Infeld action

$$
S_{B I}=\frac{1}{g_{Y M}^{2}\left(2 \pi \alpha^{\prime}\right)^{2}} \int d^{4} x \sqrt{\operatorname{det}\left(G+2 \pi \alpha^{\prime} F\right)}
$$

and perturb the open string metric by $G \rightarrow G+\kappa_{10} \epsilon$, where $\epsilon$ does not have to be symmetric.

2. Expand the Born-Infeld action (5.1) to linear level in $\epsilon$ and quadratic level in $F$ to find that

$$
\begin{aligned}
S_{B I} & =\frac{\kappa_{10}}{g_{Y M}^{2}\left(2 \pi \alpha^{\prime}\right)^{2}} \int d^{4} x \sqrt{\operatorname{det} G}\left\{\frac{1}{2} G^{\mu \nu} \epsilon_{\mu \nu}\left(1+\frac{1}{4} F_{\mu \nu} F^{\mu \nu}+\frac{1}{2} D_{\mu} \phi_{i} D^{\mu} \phi^{i}\right)\right. \\
& \left.+\frac{1}{2}\left(G^{\mu \lambda} \epsilon_{\lambda \tau} G^{\tau \nu}\right)\left(-F_{\mu \nu}+F_{\mu \rho} F_{\nu}^{\rho}-D_{\mu} \phi^{i} D_{\nu} \phi_{i}\right)\right\} .
\end{aligned}
$$

3. Fourier transform $\epsilon$

$$
\epsilon_{\mu \nu}(x)=\int \frac{d^{10} q}{(2 \pi)^{10}} \epsilon_{\mu \nu}(q) \exp \left[i q_{\mu} \cdot X^{\mu}+i q_{\perp} \cdot X\right] .
$$

4. Turn the exponential factor in (5.3) into an open Wilson line (3.7d), and at the same time integrate each operator in (5.2) along the Wilson line with respect to the $*$-multiplication and path ordering.

Following the above steps we precisely recover $(3.7 \mathrm{a})-(3.7 \mathrm{~d})$ with the identification

$$
\epsilon_{\lambda \rho} G^{\lambda \mu} G^{\rho \nu}=E^{\mu \nu}=e_{\lambda \rho}\left(\frac{1}{g+B}\right)^{\lambda \mu}\left(\frac{1}{g-B}\right)^{\rho \nu}
$$

It would be interesting to check whether higher order terms in (5.1) also reproduce the string theory amplitudes following the same steps. There are some indications that this might be true: 
- Note that the general disk amplitude (4.1) can be written as

$$
\mathcal{A}_{m}=\hat{\mathcal{A}}_{m}+\tilde{\mathcal{A}}_{m}
$$

with

$$
\begin{aligned}
& \hat{\mathcal{A}}_{m}=E \int d y Q_{m}\left(\xi_{i}, k_{i}, y_{i} ; G, \theta\right) \\
& \tilde{\mathcal{A}}_{m}=E^{M N} \int d y T_{M N}^{(m)}\left(\xi_{i}, k_{i}, y_{i} ; G, \theta\right)
\end{aligned}
$$

where

$$
E=e_{M N}\left(\frac{g-B}{g+B} D\right)^{M N}, \quad E^{M N}=e_{P Q}\left(\frac{1}{g+B}\right)^{P M}\left(\frac{1}{g-B} D\right)^{Q N} .
$$

$\hat{\mathcal{A}}_{m}$ comes from the self-contraction of $\psi$ and $\tilde{\psi}$ and $\tilde{\mathcal{A}}_{m}$ comes from the other terms. It is important to note that except for the factor $e^{i q \cdot X}$ in the closed string vertex $Q_{m}$ and $T_{M N}^{(m)}$ involve only the contractions of the open string vertex operators and are naturally expressed in terms of the open string metric $G$ and the noncommutative parameter $\theta$. The general dependence on the closed string perturbations in (5.6) is consistent with that in (5.2) with the identification (5.4).

- The additional structures (4.2) and (4.3) in the worldsheet correlation functions due to the presence of the $B$-field is consistent with the appearance of the Wilson line and the integration procedure as we outlined in Sec. .

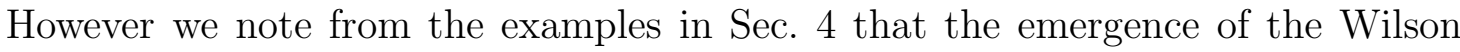
line and the integration prescription (and similarly the symmetrized trace prescription) depend crucially on the analytic properties of the worldsheet correlation functions in the low energy limit. It would be interesting to investigate them systematically. It should be helpful in understanding Matrix Theory and also D-branes in curved space.

\section{Acknowledgments}

This work was supported by DOE grant \#DE-FG02-96ER40559.

\section{References}

[1] N. Ishibashi, S. Iso, H. Kawai, Y. Kitazawa, Wilson Loops in Noncommutative Yang Mills, KEK-TH-649, KUNS-1608, hep-th/9910004. 
[2] J. Ambjorn, Y. M. Makeenko, J. Nishimura and R.J. Szabo, Finite N Matrix Models of Noncommutative Gauge Theory, J. High Energy Phys. 11 (1999) 029; [hep-th/9911041]; J. Ambjorn, Y. M. Makeenko, J. Nishimura and R.J. Szabo, Lattice Gauge Fields and Discrete Noncommutative Yang-Mills Theory, J. High Energy Phys. 05 (2000) 023; [hep-th/0004147].

[3] S. R. Das and S.-J. Rey, Open Wilson Lines in Noncommutative Gauge Theory and Tomography of Holographic Dual Supergravity, SNUST-000702, TIFR-TH/00-40, hep-th/0008042.

[4] D. J. Gross, A. Hashimoto and N. Itzhaki, Observables of Non-Commutative Gauge Theories, NSF-ITP-00-94, hep-th/0008075.

[5] N. Seiberg and E. Witten, String theory and noncommutative geometry, J. High Energy Phys. 09 (1999) 032; [hep-th/9908142].

[6] H. Liu, *-Trek II: $*_{n}$ Operations, Open Wilson Lines and the Seiberg-Witten Map, hep-th/0011125.

[7] T. Mehen and M. B. Wise, Generalized $\star$-Products, Wilson Lines and the Solution of the Seiberg-Witten Equations, hep-th/0010204.

[8] H. Liu and J. Michelson, *-TREK: The One-Loop N=4 Noncommutative SYM Action, hep-th/0008205.

[9] D. Zanon, Noncommutative perturbation in superspace, hep-th/0009196;

A. Santambrogio and D. Zanon, One-loop four-point function in noncommutative $\mathcal{N}=4$ Yang-Mills theory, hep-th/0010275.

[10] S. R. Das and S. P. Trivedi, Supergravity Couplings to Noncommutative Branes, Open Wilson Lines and Generalised Star Products,, TIFR-TH/00-52, hep-th/0011131.

[11] T. Banks, S. Seiberg and S. Shenker, Branes from Matrices, Nucl. Phys. B 490 (1997) 91-106; [hep-th/9612157].

[12] M. Li, Strings from IIB Matrices, Nucl. Phys. B 499 (1997) 149-158; [hep-th/9612222].

[13] H. Aoki, N. Ishibashi, S. Iso, H. Kawai, Y. Kitazawa and T. Tada, Noncommutative YangMills in IIB Matrix Model, Nucl. Phys. B B565 (2000) 176-192; [hep-th/9908141].

[14] N. Ishibashi, A Relation between Commutative and Noncommutative Descriptions of Dbranes, hep-th/9909176; $p$-branes from $(p-2)$-branes in the Bosonic String Theory, Nucl. Phys. B 539 (1999) 107-120, [hep-th/9804163] . 
[15] N. Seiberg, A Note on Background Independence in Noncommutative Gauge Theories, Matrix Model and Tachyon Condensation, J. High Energy Phys. 09 (2000) 003; hep-th/0008013.

[16] A. A. Tseytlin, On non-abelian generalisation of Born-Infeld action in string theory, Nucl. Phys. B 501 (1997) 41; [hep-th/9701125].

[17] R. C. Myers, Dielectric Branes,, J. High Energy Phys. 12 (1999) 022; [hep-th/9910053].

[18] W. Taylor aand M. Van Raamsdonk, Multiple D0-branes in Weakly Curved Backgrounds, Nucl. Phys. B 558 (1999) 63-95, hhep-th/9904095; Multiple Dp-branes in Weak Background Fields, Nucl. Phys. B 573 (2000) 703-734, [hep-th/9910052].

[19] H. Liu and J. Michelson, in progress.

[20] S. Hyun, Y. Kiem, S. Lee and C.-Y. Lee, Closed Strings Interacting with Noncommutative D-branes, Nucl. Phys. B 569 (2000) 262, [hep-th/9909059].

[21] M. R. Garousi, Non-commutative world-volume interactions on D-brane and Dirac-BornInfled action, IPM/P-99/051, hep-th/9909214.

[22] M. R. Garousi, Transformation of the Dirac-Born-Infeld action under the Seiberg-Witten map, hep-th/0011147.

[23] H. Liu and J. Michelson, Stretched Strings in Noncommutative Field Theory, Phys. Rev. D 62 (2000) 066003, [hep-th/0004013].

[24] D. Bigatti and L. Susskind, Magnetic Fields, Branes and Noncommutative Geometry, SUITP 99/39, KUL-TF 99/30, hep-th/9908056.

[25] M. M. Sheikh-Jabbari, Open Strings in a B-field Background as Electric Dipoles, Phys. Lett. B 455 (1999) 129-134; hhep-th/9901080].

[26] Z. Yin, A Note on Space Noncommutativity, Phys. Lett. B 466 (1999) 234-238; hep-th/9908152].

[27] M. R. Garousi and R. C. Myers, World-Volume Interactions on D-branes, Nucl. Phys. B 542 (1999) 73-88, [hep-th/9809100]; World-Volume Potentials on Branes, J. High Energy Phys. 11 (2000) 032, [hep-th/0010122].

[28] D. Kabat and W. Taylor, Linearized Supergravity from Matrix Theory, Phys. Lett. B 426 (1998) 297-305, hhep-th/9712185].

[29] A. Gerhold, J. Grimstrup, H. Grosse, L. Popp, M. Schweda and R. Wulkenhaar, The energy-momentum tensor on noncommutative spaces - some pedagogical comments, hep-th/0012112. 
[30] Y. Okawa and H. Ooguri, How Noncommutative Gauge Theories Couple to Gravity, hep-th/0012218.

[31] J. Polchinski, String Theory, Vol. I and II, (Cambridge University Press, Cambridge, England, 1998).

[32] M. Pernici, A. Santambrogio and D. Zanon, The one-loop effective action of noncommutative $\mathcal{N}=4$ super Yang-Mills is gauge invariant, hep-th/0011140.

[33] A. Hashimoto and N. Itzhaki, Non-Commutative Yang-Mills and the AdS/CFT Correspondence, Phys. Lett. B 465 (1999) 142-147; [hep-th/9907166].

[34] J. M. Maldacena and J. G. Russo, Large N Limit of Non-Commutative Gauge Theories, J. High Energy Phys. 09 (1999) 025; thep-th/9908134].

[35] N. Ishibashi, H. Kawai, Y. Kitazawa and A. Tsuchiya, A Large- $N$ Reduced Model as Superstring, Nucl. Phys. B 498 (1997) 467-491; [hep-th/9612115].

[36] T. Banks, W. Fischler, S. H. Shenker and L. Susskind, M Theory as a Matrix Model: A Conjecture, Phys. Rev. D 55 (1997) 5112-5128; [hep-th/9610043].

[37] A. Dhar, and S. R. Wadia, A Note on Gauge Invariant Operators in Noncommutative Gauge Theories and the Matrix Model, hep-th/0008144. 